\title{
Yb:YAG Innoslab amplifier: efficient high repetition rate subpicosecond pumping system for optical parametric chirped pulse amplification
}

\author{
M. Schulz, ${ }^{1,2, *}$ R. Riedel, ${ }^{2,3}$ A. Willner,,${ }^{1,2,3}$ T. Mans, ${ }^{4}$ C. Schnitzler, ${ }^{4}$ P. Russbueldt, ${ }^{5}$ J. Dolkemeyer, ${ }^{4}$ E. Seise,${ }^{3,6}$ \\ T. Gottschall,, ${ }^{3,6}$ S. Hädrich, ${ }^{3,6}$ S. Duesterer, ${ }^{1,3}$ H. Schlarb, ${ }^{1}$ J. Feldhaus, ${ }^{1}$ J. Limpert, ${ }^{3,6}$ B. Faatz, ${ }^{1,3}$ \\ A. Tünnermann, ${ }^{3,6,7}$ J. Rossbach, ${ }^{2,8}$ M. Drescher, ${ }^{2,8}$ and F. Tavella ${ }^{3}$ \\ ${ }^{1}$ Deutsches Elektronensynchrotron DESY, Notkestrasse 85, 22607 Hamburg, Germany \\ ${ }^{2}$ University Hamburg, Luruper Chaussee 149, 22761 Hamburg, Germany \\ ${ }^{3}$ Helmholtz-Institute Jena, Max-Wien-Platz 1, 07743 Jena, Germany \\ ${ }^{4}$ Amphos GmbH, Steinbachstrasse 15, 52074 Aachen, Germany \\ ${ }^{5}$ Fraunhofer Institute for Laser Technology, Steinbachstrasse 15, 52074 Aachen, Germany \\ ${ }^{6}$ Institute of Applied Physics, Friedrich Schiller University Jena, Albert-Einstein-Strasse 15, 07745 Jena, Germany \\ ${ }^{7}$ Fraunhofer Institute for Applied Optics and Precision Engineering, Albert-Einstein-Strasse 7, 07745 Jena, Germany \\ ${ }^{8}$ Center for Free Electron Laser Science, Luruper Chaussee 149, 22761 Hamburg, Germany \\ ${ }^{*}$ Corresponding author: mi.schulz@desy.de
}

Received April 18, 2011; accepted May 20, 2011; posted May 31, 2011 (Doc. ID 146164); published June 22, 2011

\begin{abstract}
We report on a Yb:YAG Innoslab laser amplifier system for generation of subpicsecond high energy pump pulses for optical parametric chirped pulse amplification (OPCPA) at high repetition rates. Pulse energies of up to $20 \mathrm{~mJ}$ (at $12.5 \mathrm{kHz}$ ) and repetition rates of up to $100 \mathrm{kHz}$ were attained with pulse durations of $830 \mathrm{fs}$ and average power in excess of $200 \mathrm{~W}$. We further investigate the possibility to use subpicosecond pulses to derive a stable continuum in a YAG crystal for OPCPA seeding. () 2011 Optical Society of America

OCIS codes: $140.4480,190.4410,190.4970$.
\end{abstract}

High repetition rate free electron lasers (FELs) [1], attosecond metrology [2], and coherent control [3] are examples of applied physics fields that require stable laser amplifier systems with very high repetition rates, high pulse energies, and ultrashort pulse durations. Free electron lasers such as FLASH would tremendously benefit when combining extreme UV (XUV) pulses and a laser amplifier with millijoule-level pulse energies, 5-20 fs pulse duration, and an intraburst repetition rate of 0.1-1 MHz to perform pump-probe experiments. Another application is the FEL seeding with similar pulse parameters [4]. Such a state-of-the-art laser system is difficult to develop, most of all because of the additional longterm stability requirements for operation at large scale facilities [5]. Optical parametric chirped pulse amplification (OPCPA) [6,7] is to date the only technique to offer a way to amplify broadband pulses at high pulse energies with several hundreds of watts average power level. An increase of the average output power of an OPCPA system requires novel concepts for the pump amplifier system. Experimental OPCPA pump amplifiers have been successfully used, either to amplify pulses at low repetition rates and high peak powers $[8,9]$ or high repetition rates and lower pulse energies $[\underline{10}, \underline{1}]$. An avenue in the multikilohertz repetition rate regime is the regenerative thin-disk amplifier that can provide millijoule pulse energy in the picosecond regime [12]. The concept has its limitations at high average powers given by difficult cavity outcoupling. Fiber chirped pulse amplification (CPA) laser amplifiers have emerged to be powerful tools for amplification to highest average powers of up to $830 \mathrm{~W}$ at femtosecond pulse durations $[13,14]$. However, combining the fiber laser amplifier with a Yb:YAG
Innoslab amplifier [15] is a promising approach to push the average power beyond the kilowatt level with multimilljoule pulse energies. A striking advantage of this amplifier combination is the attainable subpicosecond pulse duration, avoiding complicated and lossy stretcher-compressor schemes for OPCPA [16]. Recent developments have also shown the potential to use a subpicosend pump amplifier driven continuum [17] generated in bulk media to seed optical parametric amplification (OPA) [18]. Additionally, higher peak intensities can be used to drive the OPCPA system due to the inherent scaling of the damage threshold with shorter pulse duration [19]. The current study concentrates on the investigation of an Innoslab amplifier operated at a continuous repetition rate. For applications at the FEL, the amplifier will be operated in pulsed burst mode. Continuous operation at a high repetition rate will be interesting for a research and development system for dedicated studies on high harmonic generation for the FEL XUV seeding.

Previous results with a fiber CPA system for pump pulse amplification show achievable pulse energies of several tens of microjoules and sub-10-fs pulse duration at repetition rates of up to $96 \mathrm{kHz}$ using OPCPA [20]. The pump pulses were compressed in a grating compressor to pulse durations shorter than $1 \mathrm{ps}$. We recently tested

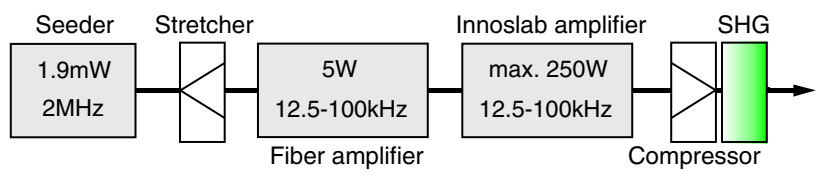

Fig. 1. Schematic setup of the pump amplifier system. 
a Yb:YAG Innoslab amplifier for further improvement of this system.

The amplifier system (Fig. 1) consists of a fiber preamplification system, delivering pulses at a center wavelength of $1030 \mathrm{~nm}$ with a bandwidth of $6.8 \mathrm{~nm}$ FWHM. The average power is $1.9 \mathrm{~mW}$ at $2 \mathrm{MHz}$ [20]. The pulses are stretched to $2.27 \mathrm{~ns}$ in an Öffner-type stretcher. The output is further amplified in a three-stage amplifier system consisting of one single mode and two large mode area photonic crystal fiber amplifiers to an average power of $5 \mathrm{~W}$. The repetition rate can be flexibly changed to subsequently seed the Innoslab amplifier [21]. The Innoslab amplifier is cw pumped by one stack of four collimated laser diode bars at a center wavelength of $940 \mathrm{~nm}$ and a power of up to $600 \mathrm{~W}$. The amplification medium consists of a longitudinally pumped Yb:YAG slab crystal with large cooled mounting surfaces for efficient heat removal. The resulting one-dimensional heat flow establishes a homogeneous cylindrical thermal lens. Two cylindrical mirrors in confocal arrangement fold the laser radiation several times through the amplification volume. The thermal lens reproduces the laser mode perpendicular to the pump line, whereas the beam is expanded in the plane of the pumped volume. In this way, the intensity increase due to the amplification is balanced. The system can be operated at repetition rates in a range from 12.5 to $100 \mathrm{kHz}$. The nominal average power for operation was $200 \mathrm{~W}$ at a $100 \mathrm{kHz}$ repetition rate. The Innoslab amplifier input spectrum of $6.8 \mathrm{~nm}$ is gain narrowed to $2.8 \mathrm{~nm}$ [Fig. 2(a)] with a Fourier-transform-limited pulse duration of $560 \mathrm{fs}$. The $M^{2}$ values of the amplified output beam (measured with a DAT-M2DU-WC with DAT-WinCamDUCD12 by Dataray Inc., second momentum method) are 1.1 in the horizontal direction and 1.4 in the vertical direction. Figure $2(\mathrm{~b})$ shows the spatial beam profile of the output beam. The highest amplified pulse energy of $20 \mathrm{~mJ}$ is attained at $12.5 \mathrm{kHz}$ and $250 \mathrm{~W}$, which was the

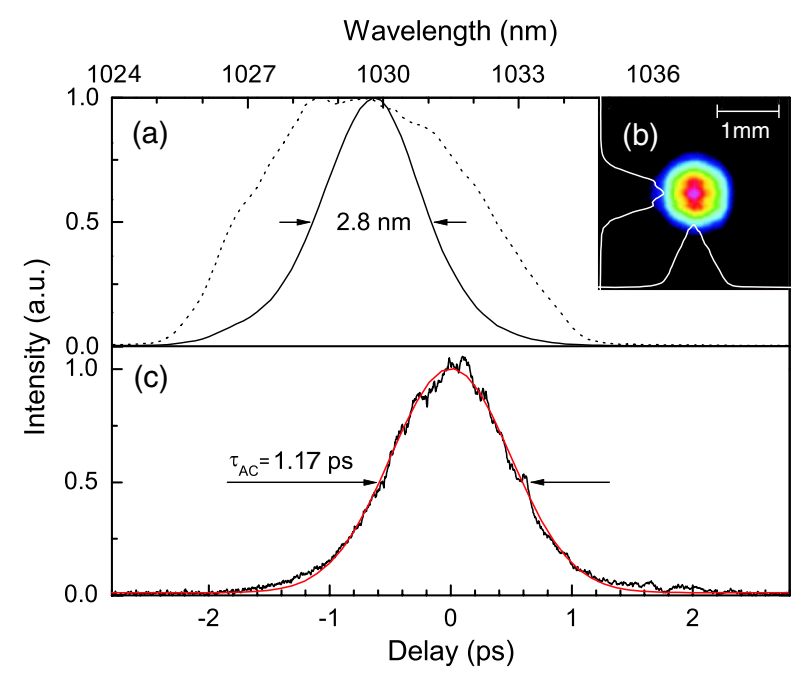

Fig. 2. (a) Input (dotted curve) and output (solid curve) pulse spectrum of the Innoslab amplifier. (b) Beam profile measurement $\left(M_{x}^{2}=1.1, M_{y}^{2}=1.4\right)$. (c) Autocorrelation measurement (black curve) and Gaussian fit (red curve) of the compressed $1030 \mathrm{~nm}$ pulses. The pulse duration corresponds to $830 \mathrm{fs}$ assuming a Gaussian temporal distribution. maximum average output power. The energy stability of the amplified pulses is measured to be $0.6 \% \mathrm{rms}$ at $100 \mathrm{kHz}$ and $1.3 \%$ at $12.5 \mathrm{kHz}$. The output power stability decreases due to the onset of $\mathrm{cw}$ self-lasing at low repetition rates below $12 \mathrm{kHz}$.

A four-pass Treacy grating compressor is used for compression of the amplified pulses. Only $160 \mathrm{~W}$ output power with the previously stated optical beam quality could be used during the compression experiment due to the lower optical quality of a spare Yb:YAG slab. The overall transmission of the compressor setup is $70 \%$ including transportation losses. The temporal characterization of the compressed pulses is performed with a (single-shot) line intensity autocorrelator [22]. Figure 2(c) shows the autocorrelation trace of the compressed pulses with an autocorrelation width of $\tau_{\mathrm{AC}}=1.17 \mathrm{ps}$ at FWHM. The resulting pulse duration is $830 \mathrm{fs}$ assuming a Gaussian temporal profile and the time-bandwidth product is 0.66 . The compressed pulses can be frequency doubled in a $1 \mathrm{~mm}$ thick type I beta-barium borate crystal with an efficiency of $57 \%$, for OPA pump generation. This leads to a total transmission-conversion efficiency of $>35 \%$ from the amplifier to the parametric amplification medium including the transportation losses.

The subpicosecond pulse duration is not only attractive for OPCPA pumping but also offers the possibility of OPCPA seed generation. So far, the longest pulse duration reported for continuum generation for seeding was $430 \mathrm{fs}$ [18]. Using longer pulses (approaching $1 \mathrm{ps}$ and above) makes a stable filamentation in bulk media difficult owing to the increasing influence of avalanche ionization $[23,24]$. A stable filament can only develop when the nonlinear self-focusing and the plasma defocusing are balanced within the peak intensity of the laser pulse [25]. For sufficient plasma defocusing, the electron density in the conduction band has to reach about $10^{18} \mathrm{~cm}^{-3}$. A rate equation was used to calculate the evolution of the electron densities created by an $850 \mathrm{fs}$ laser pulse at $1030 \mathrm{~nm}$, taking into account the amount of avalanche ionization and multiphoton excitation (MPE) [19]. The simulations show that MPE stops approximately at the peak intensity of the pulse and the generated free electrons subsequently seed the avalanche. In YAG, most of the ionization results from MPE and the electron density is kept well below the damage threshold of $10^{20} \mathrm{~cm}^{-3}$. This makes the process of continuum generation controllable and stable, contrary to the situation in sapphire, where avalanche ionization dominates and crystal damage would occur.

Experimentally, we have generated a white-light continuum in undoped YAG using pulses with a pulse energy of $10 \mu \mathrm{J}$ and a pulse duration of $850 \mathrm{fs}$, focused into a $6 \mathrm{~mm}$ long crystal (input beam diameter: $2 \mathrm{~mm}$ FWHM, $f=100 \mathrm{~mm}$ ). The focus was placed near the input surface of the crystal to achieve optimized coupling into the filament [17]. The inset in Fig. 3(a) shows the spatial beam profile. The pulse energy stability of the continuum was measured to $1.6 \%$ rms over 1 min [Fig. 3(a), Ophir PD10-PJ-SH-V2]. The spectrum of the generated continuum is shown in Fig. 3(c). It contains energy densities of 14.5 and $3.8 \mathrm{pJ} / \mathrm{nm}$ for wavelengths of 660 and $800 \mathrm{~nm}$, respectively. The spectral stability over $10 \mathrm{~min}$ is demonstrated in Fig. 3(b) (Andor Shamrock 303 spectrograph 

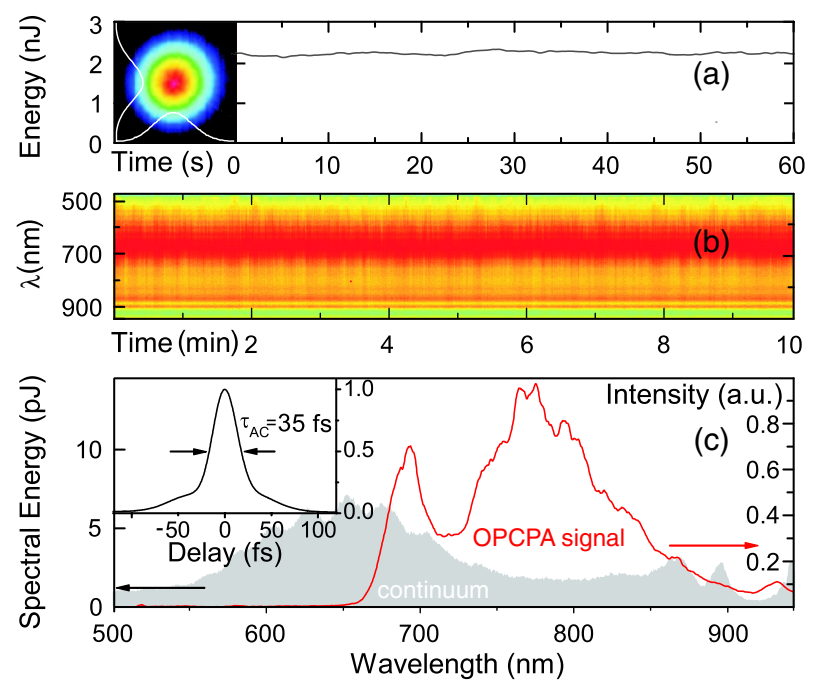

Fig. 3. (a) Spatial beam profile (inset) and energy stability of a continuum generated in an undoped YAG crystal by $10 \mu \mathrm{J}$ pulses. (b) Spectral stability over $10 \mathrm{~min}$ (logarithmic scale). (c) Spectrum of the YAG continuum (shaded gray) and spectrum of the pulses amplified in the OPCPA stage (red curve). The inset shows the AC trace of the compressed OPCPA pulses with $\tau_{\mathrm{AC}}=35 \mathrm{fs}$, corresponding to $\sim 24 \mathrm{fs}$ pulse duration.

with iDus CCD). A single stage OPCPA was set up to amplify the continuum to a pulse energy of $20 \mu \mathrm{J}$ at a $20 \mathrm{kHz}$ repetition rate. The corresponding power stability measurement shows a stability of $1.1 \% \mathrm{rms}$ (Ophir 3A-SHROHS). The amplified spectrum is plotted in Fig. 3(c) (red solid curve). The temporal compression was demonstrated using a fused silica prism pair $\left(D_{2}=-1620 \mathrm{fs}^{2}\right)$. Assuming a Gaussian temporal profile, the attained pulse duration was about $24 \mathrm{fs}\left(\tau_{\mathrm{AC}}=35 \mathrm{fs}\right)$. This was limited by strong residual negative third $\left(D_{3}=-1839 \mathrm{fs}^{3}\right)$ and fourth $\left(D_{4}=-5062 \mathrm{fs}^{4}\right)$ order dispersion. This can ultimately be compensated by adaptive dispersion control or specially designed chirped mirrors.

We have shown amplification of $1030 \mathrm{~nm}$ pulses to average powers of up to $250 \mathrm{~W}$ with maximum pulse energies of $20 \mathrm{~mJ}$ at $12.5 \mathrm{kHz}$ and $2 \mathrm{~mJ}$ at $100 \mathrm{kHz}$. The pulses are compressed to $<1 \mathrm{ps}$, which makes them interesting for OPCPA pumping. We have further investigated the possibility to use subpicosecond $1030 \mathrm{~nm}$ pulses to generate a stable continuum in a YAG crystal to seed an OPCPA stage. The continuum has a temporal stability of $1.6 \%$ rms and was used to seed a single stage OPCPA.

We thank the Fraunhofer ILT for providing the Innoslab amplifier platform and Amphos GmbH for the setup and operation of the Innoslab amplifier. We are grateful to Raphael Tautz for providing the LabVIEW program for the intensity autocorrelator. We also thank Jörg Siebenmorgen for providing the YAG crystal. This work was partially supported by the Bundesministerium fuer
Bildung und Forschung grant Förderkennzeichen 05 ES7GU1.

\section{References}

1. J. Feldhaus, J. Phys. B 43194002 (2010).

2. F. Krausz and M. Ivanov, Rev. Mod. Phys. 81, 163 (2009).

3. H. Rabitz, R. de Vivie-Riedle, M. Motzkus, and K. Kompa, Science 288, 824 (2000).

4. G. Lambert, T. Hara, D. Garzella, T. Tanikawa, M. Labat, B. Carre, H. Kitamura, T. Shintake, M. Bougeard, S. Inoue, Y. Tanaka, P. Salieres, H. Merdji, O. Chubar, O. Gobert, K. Tahara, and M.-E. Couprie, Nat. Phys. 4, 296 (2008).

5. I. Will, G. Koss, and I. Templin, Nucl. Instrum. Methods Phys. Res. A 541, 467 (2005).

6. A. Dubietis, G. Jonusauskas, and A. Piskarskas, Opt. Commun. 88, 437 (1992).

7. I. N. Ross, P. Matousek, M. Towrie, A. J. Langley, and J. L. Collier, Opt. Commun. 144, 125 (1997).

8. S. Witte, R. Th. Zinkstok, A. L. Wolf, W. Hogervorst, W. Ubachs, and K. S. E. Eikema, Opt. Express 14, 8168 (2006).

9. D. Herrmann, L. Veisz, R. Tautz, F. Tavella, K. Schmid, V. Pervak, and F. Krausz, Opt. Lett. 34, 2459 (2009).

10. S. Adachi, H. Ishii, T. Kanai, N. Ishii, A. Kosuge, and S. Watanabe, Opt. Lett. 32, 2487 (2007).

11. C. Schriever, S. Lochbrunner, P. Krok, and E. Riedle, Opt. Lett. 33, 192 (2008).

12. T. Metzger, A. Schwarz, C. Y. Teisset, D. Sutter, A. Killi, R. Kienberger, and F. Krausz, Opt. Lett. 34, 2123 (2009).

13. T. Eidam, S. Hanf, E. Seise, T. Andersen, T. Gabler, C. Wirth, T. Schreiber, J. Limpert, and A. Tünnermann, Opt. Lett. 35, 94 (2010).

14. T. Eidam, J. Rothhardt, F. Stutzki, F. Jansen, S. Hädrich, H. Carstens, C. Jauregui, J. Limpert, and A. Tünnermann, Opt. Express 19, 255 (2011).

15. P. H. Russbüldt, T. Mans, G. Rotarius, D. Hoffmann, R. Poprawe, T. Eidam, J. Limpert, and A. Tünnermann, in Advanced Solid-State Photonics, OSA Technical Digest Series (CD) (Optical Society of America, 2009), paper MF4.

16. D. M. Gaudiosi, A. L. Lytle, P. Kohl, M. M. Murnane, H. C. Kapteyn, and S. Backus, Opt. Lett. 29, 2665 (2004).

17. M. Bradler, P. Baum, and E. Riedle, Appl. Phys. B 97, 561 (2009).

18. M. Emons, A. Steinmann, T. Binhammer, G. Palmer, M. Schultze, and U. Morgner, Opt. Express 18, 1191 (2010).

19. B. C. Stuart, M. D. Feit, S. Herman, A. M. Rubenchik, B. W. Shore, and M. D. Perry, Phys. Rev. B 53, 1749 (1996).

20. F. Tavella, A. Willner, J. Rothhardt, S. Hädrich, E. Seise, S. Düsterer, T. Tschentscher, H. Schlarb, J. Feldhaus, J. Limpert, A. Tünnermann, and J. Rossbach, Opt. Express 18, 4689 (2010).

21. P. Russbueldt, T. Mans, J. Weitenberg, H. D. Hoffmann, and R. Poprawe, Opt. Lett. 35, 4169 (2010).

22. D. Herrmann, R. Tautz, F. Tavella, F. Krausz, and L. Veisz, Opt. Express 18, 4170 (2010).

23. C. B. Schaffer, A. Brodeur, and E. Mazur, Meas. Sci. Technol. 12, 1784 (2001).

24. Q. Sun, H. Asahi, Y. Yoshiaki, N. Murazawa, K. Ueno, and H. Misawa, Opt. Express 18, 24495 (2010).

25. A. Brodeur and S. L. Chin, J. Opt. Soc. Am. B 16, 637 (1999). 